\title{
Hand-Hygiene, Maintenance and Care in Dentistry, in the Age of Covid-19
}

\author{
Louis Z. G. Touyz and Sarah. J. J. Touyz
}

\section{ABSTRACT}

Dentists of necessity operate manually in the mouth. Hand hygiene is essential in the practice of modern dentistry. Eye-hand coordination demands intact healthy eyes and brain for optimal function. The oral environment can be the source of infections. This paper appraises hand hygiene, maintenance and care for practicing oral health care workers, and suggests techniques for minimizing contracting manual infections from dentistry.

Keywords: Covid-19, fungus, joints, hand, infection, hygiene, manual, nails, skin, virus, fomites, healthcare, PPE, gloves.

Published Online: October 11, 2020

ISSN: $2684-4443$

DOI : 10.24018/ejdent.2020.1.5.19

Louis Z. G. Touyz*

McGill University, Faculty of Dentistry, Qc, Canada.

(e-mail: TouyzLouis@gmail.com)

Sarah. J. J. Touyz

Salford Royal NHS Foundation Trust, UK.

*Corresponding Author

\section{INTRODUCTION AND BACKGROUND}

Dentists of necessity operate with their hands in the mouth. Hands evolved over thousands of millennia from primitive fish fins with bones, into the complex organ of tetrapods' hands. The original digital fish-fin bones are homologous to the bones found in Homo sapiens, who evolved with two hands, each with four jointed fingers and an opposing thumb [1]-[3]. The hands essentially are an extension of the brain, and hands function according to instructions from cerebral control. Functioning eyes are primarily light receptors and transmits images to the cortex that processes them and determines what reaction stimulus will be transmitted to the biomechanical constructs of the hands [3], [4]. Eye-hand coordination skills are learnt for manual operative dentistry. Accordingly, healthy functional eyes, and similarly an intact functioning neurological system pristine with a set of hands are necessary for optimal performance of dentistry. This functioning biomechanical construct and feedback loop between eyes-brain-and-hands, allows for intricate, delicate, and purposeful manipulations inherent in the practice of dental therapies. This is important as it is the main challenge for those who use prosthetic hands [4]. Yet, the mouth is not a benign working environment and houses a wide range of microbes, many of which have pathogenic potential [5], [6]. Besides the common and ubiquitous oral afflictions of both tooth decay and periodontal diseases, oral mucosal pathologies provide a wide range of microbial infections, from prions, viruses and bacteria, to unicellular organisms and parasites, all of which may reside in, or infect, the mouth [6]. Consequently all oral healthcare workers are susceptible to acquiring and spreading serious occupational diseases from dentistry, among which are hand infections. Although hand-hygiene is included in some books about infection control [7], few reports focus exclusively on prophylaxis of infection, sanitary skin-care and hygiene maintenance of oral healthcare workers' hands.
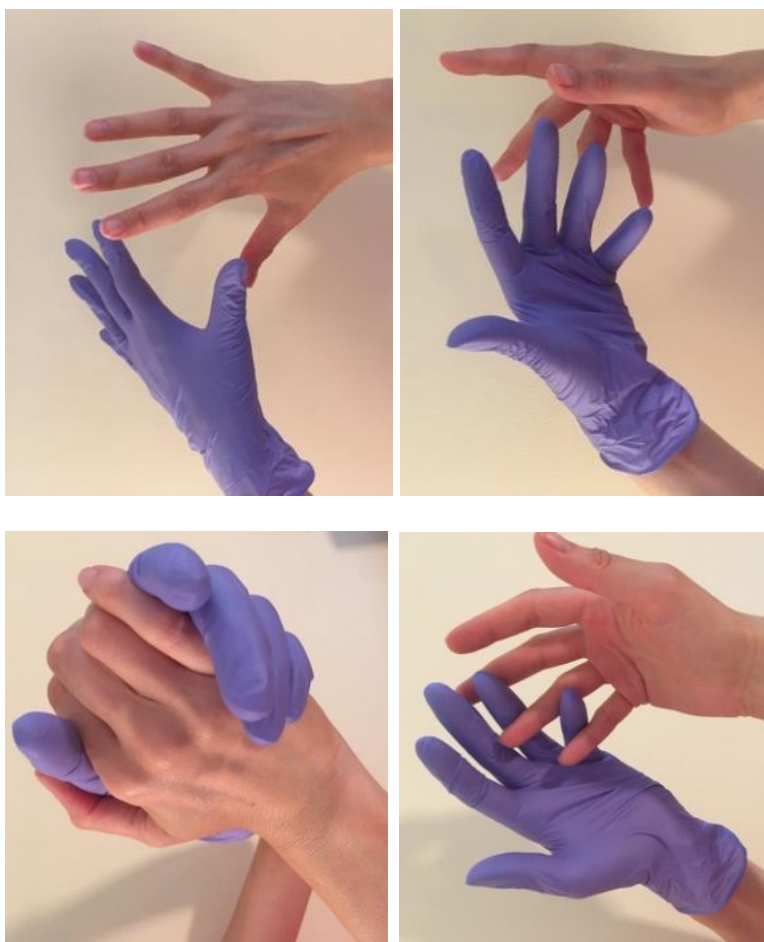

Fig. 1. The ungloved hand is not protected. Naked skin, nails and hair harbor micro- loci for infective fomites, debris, microbes, and viruses. Impervious gloves for healthcare workers protects their hands. 


\section{AIM}

This paper appraises prophylaxis against some known acquired hand infections, daily sanitizing manicure duties, long term hand-hygiene and maintenance for all oral healthcare workers.

\section{HAND PRACTICES}

Oral healthcare workers involved in dentistry include: General dental practitioners (GDP's), all oral treatment specialists, their nurses, assistants, oral hygienists and surgeons. These workers put their hands into mouths to execute exploratory, sampling or therapeutic functions. Asepsis, protection and sterilizing practices are mandatory, and demand the use of personal protective equipment (PPE) including single-use, disposable hand-gloves, gowns, eye, and masks for nasal and oral protection, as well as operatory disinfection and modern sterilizing techniques of instruments and equipment, exact details of which are described elsewhere [7]. The most important part of successfully implementing this remains the regular, if ritualized, washing of hands. Using anti-septic soap, under filtered running-water, with nailbrush scrubbing of all fingernails, is mandatory. Yet in spite of these practice measures, an ungloved or occasionally gloved hand placed into the mouth of an infected person may still unwittingly acquire an infection. Further, there always remains a risk of PPE failure and breakdown, which results in contamination and potential infection. Many cases have been reported over decades of infections picked up by oral healthcare workers.

\section{VIRAL INFECTIONS}

Common oral viral infections like Herpes simplex virus (HSV), Epstein Barr Virus (EBV), hepatitis viruses A,B,C and $\mathrm{D}$ and various human papilloma virus (HPV), or uncommon viral diseases like molluscum contangiosum, verrucae condolyma acuminatum and focal epithelial hyperplasia, have been known for decades [8]-[16]. These viruses affect the soft mucosal tissues in the mouth and are indirectly transmissible through patients' saliva carrying viable virions and infected cells, or directly transmissible into operators' minor skin abrasions.

HIV/AIDS virus have been found in saliva and exchange of bodily fluids like saliva, which can be transmitted to others [16]-[21]. Hepatitis, a DNA virus, types -A, -B, Cand $-\mathrm{D}$, are transmitted mainly from blood but can be contracted through salivary droplet spread, where miniscule amounts of blood may be present [23]. More recently, Covid-19 (a Corona RNA virus) spreads through saliva and/or coughed up mucus droplets spread through aerosolized particles expectorating, sneezing and coughing [24], [25]. Covid-19 may settle and survive on fomites and are easily spread to others, particularly susceptible people, like institutionalized people (e.g. nursing/care homes, prisons, army barracks) and in confined public indoor spaces (e.g. schools, shops, bars, restaurants, etc.). This could, and has, resulted in a massive global spread of the virus. Further, healthcare workers are particularly susceptible to infection, due to interacting with many different people and patients who may or may not manifest symptoms, or when PPE is lacking, inadequate, or deficient [26], [27].

\section{BACTERIAL INFECTIONS}

Bacterial infections are also possible through digital oral contact, especially if there are micro-abrasions on the operator's bare hands [28]. Most reports of bacterial handinfections in dentistry have been reported prior to the introduction of PPE, or in situations where PPE was not available or damaged. During oro-dental operations, sharp instruments may pierce protective gloves and allow ingress of infected blood and/ or saliva, resulting in secondary infection.

\section{FUNGAL INFECTIONS}

Chronic mucocutaneous candidiasis (CMC) is a disorder characterized by persistent and recurrent Candida species infections of the mucous membranes, particularly of the mouth and genitalia. It may be combined with various endocrine disorders (such as diabetes), and often with immune-compromised or immune-deficiency disorders (such as HIV) but can also occur in otherwise healthy individuals [29]. The skin and finger-nails are sites that are commonly affected by fungi and fungal spores as well. Prior to high and rigorous disinfection methods, Candida spores were more frequently encountered in sub-ungual sites in many ungloved oral health care workers.

Many fungal spores, besides Candida, still heavily contaminate fingernails, even after vigorous hand washing and scrubbing [32]. This highlights why it is essential to wear gloves when executing oral exams or therapy, as pathogenic fungi and bacteria may spread from fingernails and cause iatrogenic infections, and potentially infect other healthcare workers. This is a potential major cause of spread of infection, and would transmit from healthcare personnel to others, resulting in unwanted acquired nosocomial infections [33].

\section{HAIR AND HANDS}

Hair is an anlage of evolution. Accordingly, hominid apes (including orangutans, gorillas, chimpanzees and homosapiens) all have varying amounts of hair. Consequently, hair grows on the body and limbs and sometimes hair grows on the dorsal aspect of the feet and hands. Hair follicles are renown as sources for various microbes and this is why removing hair off the face (or other areas where hair grows in profusion, like the head and neck) and sometimes the arms, wrists, and hands, is essential as a pre-operation preparation to minimize sources of infection. 


\section{DISCUSSION}

\section{A. $P P E$}

Most oral healthcare workers will have their hands (usually gloved) in direct or indirect contact with saliva and blood derived from their patients, as well as the exposure to aerosolized particles generated by therapy on a patient. Should gloves be pierced during work, it is essential to immediately pause the procedure, wash and disinfect any minor wound endured, and change to a fresh pair of intact gloves. Important measures to ensure no direct tissue injury is sustained, as this would necessitate further occupational health measures to reduce the risk of having transmitted or received any potential blood borne infections; this warrants urgent medical attention. For example, needle-pricks from infected patients demand immediate care. These measures are generally covered and directed by institutional professional protocols, withs pecific guidelines and are discussed elsewhere [30], [31].

Ungloved hands would allow fungal spores (such as Candida species) to colonize the sub-ungual margins. Oral surgeons and dentists have a higher prevalence of circulating blood antibodies to Hepatitis viruses, than the general public, likely due to infection from patients [24]. In the past, despite hand washing with soap, cases of digital chancres were recorded from working with bare hands. These old, reported infections have become relics embedded in history, but are valid, good and beneficial lessons learnt, from which modern disinfection and sterilizing practices are derived [8].

\section{B. Gloves}

Hand-gloves may be made of latex rubber, but it is preferable to choose gloves made from some other nonallergenic material. Silicate baby powder is often added to facilitate donning; corn starch powder is preferable, and for both, a rinse in a bowl of sterile water pre-operatively is required.

Selecting the right size is essential; small gloves may stretch to accommodate a bigger hand, but this fatigues the hand muscles and may lead to muscular spasms, decreased sensation and dexterity during work procedures. Gloves too large produce floppy ends and interfere with tactile sensitivity needed to perform optimally. Glove-sizes should be determined pre-operatively and selections decisions finalized as part of the pre-op preparatory check lists. Gloves are manufactured in various sizes and numbered for $\mathrm{xxx}$-small number -1 up to $\mathrm{xxx}$-large number -20 . There is no universal standardization, and a trial-and-error technique is advised to select the best size and make for every operator.

\section{Hand-washing}

Hand washing is still practiced with antiseptic soaps, many containing chlorhexidine or other disinfectant chemicals. Post-washing rinsing and thorough hand-drying prevents development of "Chapped hands" and decreases the risk of any lingering microbe on the hand surfaces encountered during the procedure/examination. Surfaces wiped down with bleach or surgical alcohol $(90 \% \mathrm{Vol} / \mathrm{Vol})$ kills almost all viruses on surfaces or fomites.

Virally infected aerosols including COVID-19 are unlikely to infect people wearing adequate and properly handled PPE. Aerosolized infections are spread by close contact ( $<2$ meters) with inoculated people, many of which often don't show overt signs or symptoms of being infected. Besides PPE, social distancing is needed, and patient appointments should be punctual without any waiting to minimize contact between individuals. When doing dentistry, aerosol producing instruments like the high speed drills and ultra-sonic scalers should be eschewed. Slow rotary electric drills can be used, and manual scalers and curettes used for scaling and root-planning. It remains possible that touching infected surface and then transferring virions to the nose or mouth, will allow viral infection. Accordingly, effective autoclaves are used for successful sterilization (heat, steam and gas at $120{ }^{\circ} \mathrm{C}$ or more), for all used instruments. High level disinfectants with chemicals like dichloro-isocyanourate, bleach, gluteraldehyde and cetylpyridinium chloride solutions are widely used to disinfect apparatus which are heat labile and cannot be autoclaved, such as rubber tubing, mixing bowls, plastic spatulas, among other support ordinates.

\section{Nail hygiene}

It is desirable to keep hand - nails short, cleaned and disinfected. Sterility is desirable but unattainable in everyday use. Most people will clip their own nails, but it is desirable to have a manicurist do this at least one per month. Besides the clipping, trimming, shaping and cleaning, manicurists should check to see no undesirable infections or reactions are present. In private practice or institutions, a manicurist could visit regularly by arrangement, to do the manual care of healthcare workers. Should a nail infection or any signs of a developing 'whitlow' (sub-ungual tenderness, color change, erythema, pulp swelling, or change in sensation)be detected, the worker should be alerted and referred to a dermatologist for specialist treatment, and advised to refrain from any further work until the lesion is healed. Also, as mentioned above, hair on the forearm and usually the dorsal part of the hands, should be removed before operating (hair removal options include shaving, waxing, epilating, etc.)

\section{CONCLUSION}

Hand hygiene is very important for all manual workers, but especially healthcare workers, who need to be vigilant and disciplined about keeping their hands healthy. Transmission of disease occurs through social hand-habits, like shaking hands, and transmission of infective agents to the face, eyes and mouth will occur. Alternate social 'notouch' greeting habits like bowing seems preferable to handshakes. It is essential to wash hands frequently after daily chores and ablutions, to minimize introducing or transmitting infections. 


\section{REFERENCES}

[1] Long J. L., Cloutier R. (2020) The unexpected origin of fingers. Sci Amer. June; 322; 6: 46-53.

[2] Cloutier R. (2020) Elpistostege and the origin of the vertebrate hand. Nature. June 2020. 579; 549-554.

[3] Rohen J. W., Yokochin C., Lütjen-Drecol. (2006) In Color Atlas of Anatomy. 6thEd.Ch 7. Upper limb.422-430Fore arm and lower limb. The HAND. 2006. Schattauer Stuttgart; ct Wiliams and Wilkins New York.

[4] Ferreri F., Ponzo D., Vollero L., Petrichelli S., et al (2014) Does an intraneural interface short-term implant for robotic hand contro modulate sensorimotor cortical integration? An EEG-TMS coreistration study on a human amputee. Jnl Neurology and Neuroscience. 2014. 32:2; 281-292.

[5] Touyz L. Z. G (2017) The Pathophysiology of Oral Biofilms and its' relation to Initial Gum Disease and Caries. J Dent Oral Disord Ther 5(4):1-6. Symbiosis Group.

[6] Laskars G. ((1988) Color Atlas of Oral Disease. Passim, Thiemerlag, Stuttgat; NewYork.

[7] Miller C H. (2018) In: Infection Control. 6thEd Ch 11. 94-100. Hand Hygiene: Hands and disease spread p94.-5; Hand hygiene agents 95. Elsevier.

[8] Touyz L. Z. G. (2020) Antiseptic, Aseptic and Sterilizing Practices in General Dentistry In the Age of COVID-19. An Appraisal. J Oral health Dent res. 2020:1(2); 1-5.

[9] Spruance SL (1995). In Clinical Management of Herpes Viruses. Herpes simplex labialis. Sacks S L, Straus S E, Whitley R J, Griffiths P D Eds. Amsterdam IOS Press. p 5-6.

[10] Spruance S. L., Overall J. C., Kern E. R, Krueger G. G., Pliam V., Miller W, (1977) The natural history of recurrent Herpes simplex labialis: implications of antiviral therapy. N Eng Jnl Med; 297(2), 6975.

[11] Spruance S. L. (1995). In Clinical Management of Herpes Viruses. Herpes simplex labialis. Sacks SL, Straus SE, Whitley RJ, Griffiths PD Eds. Amsterdam IOS Press. p 5-6.

[12] Raborn G. W., Grace M. G. A. (2003) Recurrent Herpes Labialis: Selected Therapeutic options. J Can Dent Assoc 69(8):498-503.

[13] Burn J. C. (1980) Diagnostic methods for Herpes simplex infection. A review. Oral Surg 50: 346-341.

[14] Courant P. (1969) Oral manifestations of Infectious Mononucleosis. J Periodontol 40:779-784

[15] Slots J., Saygun I., Sabeti M., Kubar A. (2006) Ebstein-Barr Viruses in Oral Diseases. J Perio Res 41:235-242.

[16] Touyz L. Z. G. (2013) Human Papilloma Virus (HPV)-A Biological and Clinical Appraisal: 2013. Science Postprint Japan. Human Papilloma Virus (HPV)-A Biological and Clinical Appraisal: 2013. Science Postprint 1(1): e00001. doi:10.14340/spp.2013.10R0002.

[17] Clausen F. P. (1972. Rare oral viral disorders. (molluscum contangiosum, localized keratoacanthoma, verrucae, condyloma acuminatum, and local epithelial hyperplasia. Oral Surg 34: 604-609.

[18] Rose L. F., Mealy B. L. (2004) In: Periodontics. Part V Ch31 HIV \& AIDS. 818-831.

[19] AnnerothG., Anneroth L., Lynch D.P. (1986) Acquired immunodeficiency Syndrome (AIDS) in the United States to 1986.Etiology, epidemiology, clinical manifestations and dental implications. J Oral Maxillofac Surg. 42: 956-965.

[20] 21.Mardirossian A., Contreras A., Navazesh M., Nowzar H., Slots J. (2000) Herpes virus 6,7 and 8 in HIV- and non-HIV associated Periodontitis. J Periodontol Res 35: 278-284.

[21] Ling L-J., Ho C-C., Wu C-Y. et al (2004) Association between HSV and severity of periodontitis. $J$ Periodontol 75;1479-1485.

[22] Solomon E. P., Berg L. R., Martin D. (2005) In: Biology. 7th Ed. Ch 23; Viruses \& Prokaryotes 435-439. Thompson Brooks/Cole.

[23] Slots J., Saygun I., Sabeti M., Kubar A. (2006) Ebstein-Barr Viruses in Oral Diseases. J Perio Res, 41:235-242.

[24] Mahboobi M., Agha-Hosseini F., Mahboobi N., et al (2010) Hepatitis B virus infection in Dentistry: a forgotten topic. Journal of Viral Hepatitis. May 2010. 17:5:307-316.

[25] Norkin L. C. (2010) In "Virology" Molecular Biology and Pathogenesis. 2010. Ch3 Modes of Virus Infection and disease. 50-78. ASM Press Washington DC, USA.

[26] Qiu J. (2020) Chasing plagues; origins of the first SARS virus and the current pandemic. Si Amer. June 2020. 322:6; 26-32.

[27] Waldholz M. (2020) Fast track Drugs. Existing compound that deflect harm. Sci Amer, June 2020. 322:6; 32-35.
[28] Mock J. (2020) Frontline trauma; Medical workers are societys'new heroes. What happens when the acute crisis is over. Sci Amer. June 2020. 322:6; 36-37

[29] Liu X, Hua H. (2007) Oral manifestations of chronic mucocutaneous candidiasis. Seven Case reports. Jnl Otral Path and Med. 36:9: 528 523.

[30] Regez R., M., Rietra P. J. G. M., van der Linden Th., M,.et al. (2002) reducing the risk of blood-transmitted infections of HIV, Hepatitis B or $\mathrm{C}$, in a teaching hospital in Amsterdam - evaluation of a protoco for needle-stick accidents among Hospital staff during the period 1997-2001. Ned TijschrGeneedskd. Mar 2002. 30; 146 (13): 617-621.

[31] Mahmood H., Awan J., Chaudry A. (2018) Frequency of Needlestick Injury among Dental care Personnel in Islamabad dental Hospital Pakistan Oral and Dental Journal. Periodontics. April-Jun 2018. 38:2:

[32] Editorial- The Lancet: Teenagers with adult Diseases. The Lancet 371. 2008. 9617.960.

[33] Sohoni R., Koshi A. V., Bhaitia V. M., et al (2018) The incidence and Nature of fingernail Flora after routine Pre-operative Hand Scrubbing. Int Jnl Prev and Clin Dent res. April 1018:5(2)" 56-58.

[34] Donovan B. (2004) Sexually transmitted infections other than HIV. The Lancet 363.2004: 9408.p.545-556. 